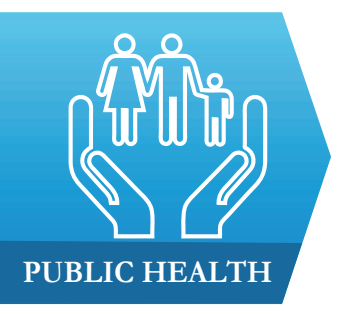

1) Department of Analytical Chemistry and Instrumental Analysis, Faculty of Pharmacy, Iuliu Hatieganu University of Medicine and Pharmacy, Cluj-Napoca, Romania

2) Department of Drug Information Research Centre, Iuliu Hatieganu University of Medicine and Pharmacy, Cluj-Napoca, Romania

DOI: $10.15386 / \mathrm{mpr}-1201$

Manuscript received: 02.10 .2018

Received in revised form: 28.01.2019

Accepted: 12.02.2019

Address for correspondence:

Laura.Anghel@umfcluj.ro

\section{An overview of the common methods used to measure treatment adherence}

\author{
Laura Alexandra Anghel ${ }^{1}$, Andreea Maria Farcas ${ }^{2}$, Radu Nicolae Oprean ${ }^{1}$
}

\begin{abstract}
Background and aims. The success of a treatment depends on the effectiveness of the medication regimen, provided that patients take the medicines as prescribed. A low rate of adherence in chronic conditions is associated with poor outcome and decreased quality of life, which constitutes an additional burden for the healthcare systems. To correctly identify the dimension of this problem may be a challenge, as there are numerous methods, definitions, patient settings and factors, each with their specific roles. Our aim was to give an appropriate overview of the most common validated methods that can be used to identify non-adherent patients.

Methods. This overview is based on an online search of PubMed database and includes the relevant articles in this field.

Results. We included both direct and indirect methods for measuring treatment adherence and presented concise information that can help researchers and clinicians when choosing an appropriate method. Both subjective and objective methods have advantages and disadvantages that should be fully understood and taken into consideration.
\end{abstract}

Conclusions. Choosing a simple, accurate and inexpensive method that can give supplementary information about the patterns, beliefs and barriers of adherence would be desirable. But because this perfect method to measure treatment adherence does not exist, the best solution seems to be the combined use of at least two methods.

Keywords: patient, adherence, drug therapy, chronic diseases

\section{Background}

Optimal treatment adherence is essential for the management of chronic conditions and the effectiveness of prescribed therapies. A World Health Organisation (WHO) report underlines the fact that adherence to chronic treatments is as low as $50 \%$ [1]. More recent data describe low-adherence as being a "silent epidemic", which is likely to play a role in $21-37 \%$ of preventable adverse drug events [2]. The implications of low adherence translate into negative outcomes, such as increased morbidity and mortality and supplementary cost for healthcare systems. This has been repeatedly demonstrated for a number of chronic conditions: asthma, diabetes, tuberculosis, cardiovascular disorders, HIV, mental disorders, rheumatic conditions [1-8].

Being able to correctly identify and estimate treatment adherence has become a research focus in many chronic conditions, and the importance of increasing the effectiveness of adherence interventions is considered to "have a far greater impact on the health of the population than any improvement in specific medical treatments" $[1,8]$. However, effective interventions to improve treatment adherence can only be developed if research can also provide additional information about predictors and factors related to adherence, for a better understanding of the individual causes.

Treatment adherence continues to be an incredibly complex subject. This is associated with the terminology used (terms like compliance, persistence, discontinuation are still being used as synonyms or equivalents to adherence), the multitude of measurements methods that exist and the vast amount of factors and predictors influencing adherence that have been studied $[9,10]$.

One of the first terms introduced was "compliance", and was defined as 
"the extent to which a patient acts in accordance with the prescribed interval and dose of a dosing regimen". This was viewed as having the negative connotation that patients are obedient to healthcare providers (HCP) instructions. As it is viewed today, the act of prescribing a treatment is based on a shared decision between patient and HCP, thus both have the ability to influence medicine taking behavior [11-13]. The current definition of treatment adherence, as given by the WHO, is "the extent to which a person's behavior- taking medication, following a diet, and/or executing lifestyle changes- corresponds with the agreed recommendations from a healthcare provider" [1]. Medication persistence is another concept related to adherence, and refers to the pursuing of treatment for the prescribed duration, and is defined as being " the duration of time from initiation to discontinuation of therapy" $[12,14,15]$.

Different tools and measurements have been developed and validated in order to effectively and accurately assess adherence and persistence in a wide range of diseases. Each has advantages and disadvantages that should be thoroughly taken into consideration when designing and choosing a suitable method.

\section{Aims}

To write an up-to-date overview of the validated methods most frequently used to measure treatment adherence, in order to give researchers and clinicians basic guidance in choosing the most appropriate method.

\section{Methods}

We conducted a literature search on the PubMed database limited to articles published in English. Relevant articles (reviews, reports, opinions) were included, based on screening of the abstract or/and full text and their importance in the field. We considered that an extended review on this subject may prove to be excessive, since the purpose was to offer a summary of the available methods used to measure treatment adherence and for this reason we selected representative articles to be included in this research.

\section{Results}

Different approaches have been developed and validated to be used in various conditions and settings. Generally they are divided into subjective and objective methods, and in addition they can also be classified into direct and indirect methods.

Adherence is usually measured over a period of time and reported as a percentage, offering information about dose taking behavior in relation to what was prescribed. In some cases adherence is reported as dichotomous variable (adherent/ non-adherent) or classifying levels of adherence (low/high adherers) and even as a mean value.

Maintaining a treatment regimen (continuing to take any amount of medication) is mainly consistent with the definition of persistence. Persistence is expressed as a continuous variable, reporting the number of days for which treatment was available. Sometimes even as a dichotomous variable or as an average for the entire study period.

A brief overview of the methods available to measure different aspects of treatment adherence, including advantages and disadvantages, as well as parameters that are measured, are presented in Table I.

Table I. Adherence methods.

\begin{tabular}{|c|c|c|c|}
\hline Methods of assessment & Advantages & Disadvantages & Parameter measured \\
\hline \multicolumn{4}{|l|}{ Direct } \\
\hline $\begin{array}{l}\text { Measurement of drug/ } \\
\text { metabolite levels }\end{array}$ & $\begin{array}{l}\text { Accurate } \\
\text { Objective, proving the } \\
\text { ingestion of the drug }\end{array}$ & $\begin{array}{l}\text { Costly } \\
\text { Invasive } \\
\text { Inter individual differences }\end{array}$ & Concentration of the drug/metabolite \\
\hline \multicolumn{4}{|l|}{ Indirect } \\
\hline Pill counts & $\begin{array}{l}\text { Simple } \\
\text { Mostly used in clinical trials }\end{array}$ & $\begin{array}{l}\text { No evidence of ingested } \\
\text { medication }\end{array}$ & Number of doses missed \\
\hline Electronic databases & $\begin{array}{l}\text { Easy to use } \\
\text { Inexpensive } \\
\text { Non-invasive, patients not } \\
\text { aware that they are being } \\
\text { monitored } \\
\text { Especially specific to identify } \\
\text { non-adherent patients }\end{array}$ & $\begin{array}{l}\text { Evidence of the drug being } \\
\text { dispensed but not ingested }\end{array}$ & $\begin{array}{l}\text { Medication possession ration (MPR) } \\
\text { Proportion of days covered (PDC) }\end{array}$ \\
\hline $\begin{array}{l}\text { Self-reported } \\
\text { (questionnaires, visual } \\
\text { analogue scales) }\end{array}$ & $\begin{array}{l}\text { Easy to use } \\
\text { Inexpensive }\end{array}$ & $\begin{array}{l}\text { Overestimate adherence } \\
\text { Subjective, influenced by } \\
\text { recall or reporting bias }\end{array}$ & $\begin{array}{l}\text { A value that is interpreted in regards } \\
\text { to a pre-established cut-off point }\end{array}$ \\
\hline $\begin{array}{l}\text { Electronic monitoring } \\
\text { systems }\end{array}$ & $\begin{array}{l}\text { Objective } \\
\text { Additional information on } \\
\text { degree of adherence } \\
\text { One of the most accurate } \\
\text { methods }\end{array}$ & $\begin{array}{l}\text { The patient is aware of the } \\
\text { evaluation } \\
\text { No actual evidence that the } \\
\text { medication is being ingested }\end{array}$ & $\begin{array}{l}\text { Overall percentage of doses taken } \\
\text { Dosing regime }\end{array}$ \\
\hline
\end{tabular}




\section{Discussion}

The commonly used classification of the adherence assessment methods, used by the WHO, refers first of all to subjective and objective methods. Subjective methods involve the patient's assessment of their medication taking behavior or healthcare provider (usually with the help of a questionnaire) and they are inclined to a certain degree of bias. On the other hand, objective methods (such as measurements of clinical outcomes, dose counts, pharmacy records, electronic monitoring of medication administration) have the apparent potential to best measure treatment adherence. Further classification refers to direct and indirect methods of assessment [16]. Direct methods refer to the direct observation of therapy or measurement of the drug (or metabolite) or a biological markers levels in blood or urine, that prove that the medication has been taken by the patient. Indirect methods involve patient questionnaires, patient self reports, pill counts, rates of prescription refills, assessment of patient's clinical response, electronic medication monitors, measurement of physiologic markers, or patient diaries. We will further discuss each of the methods, underlining their specifics advantages and disadvantages.

\section{Direct methods}

Direct methods include measurements of the drug (or a metabolite) concentration in body fluids. Although it may be considered as being an adequate and precise method, which can offer strong evidence of the ingestion of the drug, there are some variables that should be considered. Drug metabolism, individual variation in the pharmacokinetics of the drug, drug-drug interactions and drug-food interactions may interfere with the accuracy of the method, making it inapplicable for some drugs that have long halving time and can be detected long after the patient has stopped the treatment. The method is appropriate for the measurement of adherence to one drug therapy regimen only and it offers no supplementary data on the additional causes of non-adherence and does not report on any patterns of nonadherence. It is also quite expensive, and could be viewed as interventional by some patients. Potential bias should also be considered, with some patients beginning taking their medication before upcoming examinations. The parameter measured, the presence of the drug (or metabolite), simply generates a yes/no result. Therefore this method does not offer additional information regarding patterns or levels of adherence or factors that could influence it [17-19].

The direct observation of the patient's medicationtaking behavior by healthcare practitioners (HCP) can provide proof of the ingestion of the medicine, but in some cases can be impractical and not entirely accurate (patients can mimic the administration of the medicine, only to discard it afterwards) Moreover, direct observations are possible only for hospitalized or institutionalized patients and impractical to use in large population settings. Bias can easily be introduced if patients administer the medication only when they receive visits from the $\mathrm{HCP}$, leading to false adherence [17-19].

\section{Indirect methods}

Indirect methods are by far more popular in adherence research, and include: pill count, electronic monitoring devices, the use of electronic health records, and self reported measures.

Pill count is a simple method that calculates the number of doses that have been taken between appointments, and compares it with the total number of doses that the patient has received. An adherence ratio is then calculated. This is a straightforward and low cost method that can be easily applied for different type of formulations (tablets, inhalers). It can assess an average adherence, but does not give specific information about daily adherence or patterns of adherence. It is based on the assumption that removing the correct number of tablets from the dosing unit is equivalent with taking the medicine as recommended. However, this method does not prove actual ingestion of the drug. Potential variations in the dosing regimen should also be considered, especially in chronic conditions [17-19].

Measuring adherence and /or persistence using electronic databases (pharmacy and insurance claim databases, registries), is based on the assumption that prescription refilling patterns coincides with medicationtaking behavior. This requires the existence of a centralized, electronic system with consistency among prescribers and dispensers, and can be a convenient and inexpensive method to measure adherence $[14,15,20]$. Prescription refill data has the capacity to provide rough adherence estimations, since it offers information on the possession of medication and not proof on actual intake of medicine, and in some cases could give overestimations. It gives the opportunity to assess non-adherence in a large population over an extensive period of time, including multi-drug non-adherence. This method has the down-side of not being able to give very much information regarding barriers or factors associated with non-adherence (besides demographic features). By studying registries, we can obtain valuable data on causes of discontinuation (like ineffectiveness, suspected adverse drug reactions), since most registries give accurate information about different aspects of the patient treatment regimen [17-20]. However, it does not give information on individual patients' rates of adherence, being mostly used for research purposes. Pharmacy records are accurate, inexpensive and easy and practical for adherence and persistent assessment.

The two, most commonly, measured parameters in pharmacy claim databases are Medication Possession Ratio (MPR) and Proportion of Days Covered (PDC). MPR is frequently defined as "the proportion (or percentage) of days supply obtained during a specified time period or over a period of refill intervals". When interpreting and analyzing data from literature it is important to assess how the MPR was calculated, since there are various methods 
for MPR calculation. PDC is the number of days when the drug was available divided by the number of days in the study period. A cut-off point is commonly advised (at least $80 \%$ adherent), which categorizes the patients as being adherent and non-adherent $[21,22]$. Some studies even use this as a continuous variable.

Electronic monitoring devices, such as Medication Event Monitoring System (MEMS) are devices incorporated in the container that stores the dosing history of the patient's prescribed medication. The opening of the container is assumed to coincide with the ingestion of the medication. It has been proven to be highly accurate in several studies, and it is used as a reference standard for validating other adherence methods [17,19,23-25]. It gives precise and detailed information about the number of doses taken and other deviations from the dosing regimen. Incorrect use of the device and opening the container without taking the medication could lead to false results [23-25]. The use of MEMS in large populations is limited by the relative high price of the device, as well as some practical issues like potential complications that may arise with refilling the prescription in the local pharmacy or some medication formulations.

Self-reported methods are among the most cheap and simple procedures for measuring adherence. Whether they are distributed online, administrated as structured interviews, or written questionnaires they enjoy a high degree of popularity in adherence research and can easily be adapted in different patient populations $[15,18,19]$. They are generally perceived as tending to overestimate adherence, in comparison to direct methods, since the patients can be influenced by recall or reporting bias.

Questionnaires are a standardized self-reported measure for evaluating adherence to a specific medication regimen and can also give additional information about attitudes, behaviors and intentions. They are customarily validated against other measures, and a large number have been also strongly correlated with objective measures in different patients populations $[25,26]$. A cut-off point is chosen (commonly at $80 \%$, thought in some studies this can be as low as $60 \%$ ) to differentiate between adherent and non-adherent patients.

Depending on the type of information that they seek to collect, they can be grouped into questionnaires that assess:

- Only medication-taking behavior

- Medication-taking behavior and barriers to adherence

- Only barriers to adherence

- Only beliefs associated with adherence

- Barriers and beliefs associated with adherence [26].

According to this classification some of the most commonly used adherence scales fall into distinct categories, and attention is recommended both, when choosing and, when interpreting results from different studies. We will briefly discuss hereby the most frequently used scales: Medication Adherence Report Scale (MARS), The Compliance Questionnaire Rheumatology (CQR19) and Belief about Medicines Questionnaire (BMQ).

The first variant of the Medication Adherence Report Scale (MARS), developed in 2000, included 10 statements, with a yes/no answer, validated to be used for patients with schizophrenia. It is based on Drug Attitude Inventory (DAI) and the MAQ and assesses beliefs and barriers to medication adherence general disease control during the past week [26,27].

The MARS-5 and 6 are variations of this, including five, respectively six statements with a five point rating scale, designed to be used in different chronic conditions (asthma, chronic obstructive pulmonary disease, diabetes, rheumatoid arthritis). Easy to apply, MARS is frequently used in adherence research $[28,29]$.

The Compliance Questionnaire Rheumatology (CQR19) is the only questionnaire specifically developed and validated (against MEMS device) to be used in patients with inflammatory rheumatic diseases. The 19 items are scored on a 4-point Likert response scale from 1 to 4 , and the score ranges from 0 (complete non-compliance) to 100 (perfect compliance) [26,30]. A cut-off point is usually advised when using this scale, but in some cases it is also used as a continuous variable. CQR19-describes barriers and beliefs and was identified to offer information only in the implementation phase, making it to be more appropriate for use in first-time users. In an attempt to make it more easy to incorporate in clinical practice, a five items questionnaire was derived from this, maintaining a good level of reliability and validity [26,31].

The Beliefs about Medicines Questionnaire (BMQ) is a good example of a scale focused on measuring the specifics of treatment adherence-behavior rather than a pure quantification of it. It is indented to be used with another method of measuring adherence and it gives supplementary information about the general and specific necessity beliefs and concerns by assessing cognitive representations of medication. The questionnaire has been developed based on identified beliefs in literature which appeared to be common to patients with a wide range of chronic conditions, and based on interviews with patients undergoing drug treatment for chronic conditions. The complete version of the BMQ is composed of two sections: the General section (BMQ-General 18 items), which assesses more general beliefs about medicines and the Specific section (BMQSpecific 16 items), which explores beliefs about medication prescribed for personal use. The two sections (with a total of 34 statements, which can be administered within 10 minutes) can be used in combination or separately. The BMQ has been translated and adapted to be used in several countries and validated in patients with asthma, diabetes, psychiatric and cardiac disorders [32,33]. All items have a five-point Likert scale answer option, which varies from 
strongly agree to strongly disagree. Higher scores indicate stronger beliefs about the corresponding concepts in each sub-scale.

The short administration time makes the BMQSpecific a potentially applicable instrument in everyday clinical practice. The latest drug adherence research underlined the major impact of personal beliefs and concerns that arise in patients. They can explain much better the individual variations of drug adherence behavior than socio demographic and clinical factors [34-37]. By such, identifying personal drivers of adherence can contribute to the development of effective and special tailored interventions to improve adherence.

For a method to be ideal in adherence measurement it should be: easy to apply in any setting, accurate and not expensive, and able to provide additional information regarding potential barriers, beliefs or concerns that patients might have. The choice should be also based on the response burden of the patient, and how the final results will be used. However, until now, there is no such method, as seen above, each method comes with advantages and disadvantaged that limits their use in some cases. For that reason, a multimodal approach seems to be the most appropriate solution. Using two (or if possible more) methods can offer more accurate and complex information about treatment adherence and can also confirm the original findings as seen in recent studies $[1,28,37]$.

\section{Conclusions}

This paper should provide a general direction for professionals when choosing an adequate method in their adherence research and while this is not a comprehensive review of all the methods used in adherence research, it gives information on various types and the most frequently accepted methods that can be applied in different patients' settings.

Since there is no method that is regarded as "gold standard" in evaluating adherence, selecting at least two methods can give results that are closer to reality: an objective method that gives solid proof of the ingestion of medication and a subjective method that completes the research with information regarding factors, beliefs or barrier of adherence.

\section{References}

1. World Health Organisation. Adherence to long-term therapies: evidence for action. Available from : http:/www.who.int/chp/ knowledge/publications/adherence_report/en/

2. Uppsala Monitoring Centre. Better Pharmacovigilance for All. Uppsala Reports. 2017;(76). Available from: https://view.publitas. com/uppsala-monitoring-centre/uppsala-reports-76_web/page/1

3. Kennedy-Martin T, Boye KS, Peng X. Cost of medication adherence and persistence in type 2 diabetes mellitus: a literature review. Patient Prefer Adherence. 2017;11:1103-1117.

4. Bereza BG, Troelsgaard Nielsen A, Valgardsson S, Hemels ME, Einarson TR. Patient preferences in severe COPD and asthma: a comprehensive literature review. Int J Chron Obstruct Pulmon Dis. 2015;10:739-744..

5. Deshpande S, Quek RG, Forbes CA, de Kock S, Kleijnen J, Gandra SR, et al. A systematic review to assess adherence and persistence with statins. Curr Med Res Opin. 2017;33:769-778. 6. Shubber Z, Mills EJ, Nachega JB, Vreeman R, Freitas M, Bock $\mathrm{P}$, et al. Patient-Reported Barriers to Adherence to Antiretroviral Therapy: A Systematic Review and Meta-Analysis. PloS Med. 2016;13:e1002183.

7. Smith D, Lovell J, Weller C, Kennedy B, Winbolt M, Young $\mathrm{C}$, et al. A systematic review of medication non-adherence in persons with dementia or cognitive impairment. PLoS One. 2017; 12:e0170651.

8. Iuga AO, McGuire MJ. Adherence and health care costs. Risk Manag Healthc Policy. 2014; 7:35-44.

9. Horne R, Chapman SC, Parham R, Freemantle N, Forbes A, Cooper V. Understanding patients' adherence-related beliefs about medicines prescribed for long-term conditions: a metaanalytic review of the Necessity-Concern Framework. PLoS One. 2013;8:e80633.

10. Haynes RB, McDonald H, Garg AX, Montague P. Interventions for helping patients to follow prescriptions for medications. Cochrane Database Syst Rev. 2002;(2):CD000011.

11. Fraser S. Concordance, compliance, preference or adherence. Patient Prefer Adherence. 2010;4:95-96.

12. Cramer JA, Roy A, Burrell A, Fairchild CJ, Fuldeore MJ, Ollendorf DA, et al. Medication compliance and persistence: terminology and definitions. Value Health. 2008;11:44-47.

13. Vrijens B, De Geest S, Hughes DA, Przemyslaw K, Demonceau J, Ruppar T, et al. A new taxonomy for describing and defining adherence to medications. Br J Clin Pharmacol. 2012;73:691-705.

14. Sikka R, Xia F, Aubert RE. Estimating medication persistency using administrative claims data. Am J Manag Care. 2005;11:449457.

15. Forbes CA, Deshpande S, Sorio-Vilela F, Kutikova L, Duffy S, Gouni-Berthold I, et al. A systematic literature review comparing methods for the measurement of patient persistence and adherence.Curr Med Res Opin. 2018;34:1613-1625.

16. Osterberg L, Blaschke T. Adherence to Medication. N Engl J Med. 2005;353:487-497.

17. Lam WY, Fresco P. Medication Adherence Measures: An Overview. Biomed Res Int. 2015;2015:217047.

18. De Achaval S, Suarez-Almazor ME. Treatment adherence to disease-modifying antirheumatic drugs in patients with rheumatoid arthritis and systemic lupus erythematosus. Int J Clin Rheumatol. 2010;5:313-326.

19. Van den Bemt B, Zwikker HE, van den Ende CH. Medication adherence in patients with rheumatoid arthritis: a critical appraisal of the existing literature. Expert Rev Clin Immunol. 2012;8:337351.

20. Raebel MA, Schmittdiel J, Karter AJ, Konieczny JL, Steiner JF. Standardizing terminology and definitions of medication adherence and persistence in research employing electronic databases. Med Care. 2013;51(8 Suppl 3):S11-S21.

21. Bonafede M, Johnson BH, Tang DH, Shah N, Harrison DJ, Collier DH. Etanercept-Methotrexate Combination Therapy Initiators Have Greater Adherence and Persistence Than Triple Therapy Initiators With Rheumatoid Arthritis. Arthritis Care Res (Hoboken). 2015;67:1656-1663.

22. Chu LH, Kawatkar AA, Gabriel SE. Medication adherence 
and attrition to biologic treatment in rheumatoid arthritis patients. Clin Ther. 2015;37:660-666.e8.

23. Checchi KD, Huybrechts KF, Avorn J, Kesselheim AS. Electronic medication packaging devices and medication adherence: a systematic review. JAMA. 2014;312:1237-1247.

24. El Alili M, Vrijens B, Demonceau J, Evers SM, Hiligsmann M. A scoping review of studies comparing the medication event monitoring system (MEMS) with alternative methods for measuring medication adherence. $\mathrm{Br} \mathrm{J}$ Clin Pharmacol. 2016;82:268-279.

25. Shi L, Liu J, Fonseca V, Walker P, Kalsekar A, Pawaskar M. Correlation between adherence rates measured by MEMS and self-reported questionnaires: a meta-analysis. Health Qual Life Outcomes. 2010;13;8:99.

26. Nguyen TM, La Caze A, Cottrell N. What are validated selfreport adherence scales really measuring?: a systematic review. Br J Clin Pharmacol. 2014;77:427-445.

27. Thompson K, Kulkarni J, Sergejew AA. Reliability and validity of a new Medication Adherence Rating Scale (MARS) for the psychoses. Schizophr Res. 2000;42:241-247.

28. De Cuyper E, De Gucht V, Maes S, Van Camp Y, De Clerck LS. Determinants of methotrexate adherence in rheumatoid arthritis patients. Clin Rheumatol. 2016;35:1335-1339.

29. Kumar K, Raza K, Nightingale P, Horne R, Chapman $\mathrm{S}$, Greenfield $\mathrm{S}$, et al. Determinants of adherence to disease modifying anti-rheumatic drugs in White British and South Asian patients with rheumatoid arthritis: a cross sectional study. BMC Musculoskelet Disord. 2015;16:396.

30. de Klerk E, van der Heijde D, van der Tempel H, van der Linden S. Development of a questionnaire to investigate patient compliance with antirheumatic drug therapy. J Rheumatol. 1999;26:2635-2641.

31. Hughes LD, Done J, Young A. A 5 item version of the Compliance Questionnaire for Rheumatology (CQR5) successfully identifies low adherence to DMARDs. BMC Musculoskelet Disord. 2013;14:286.

32. Horne R, Weinman J, Hankins M. The beliefs about medicines questionnaire: the development and evaluation of a new method for assessing the cognitive representation of medication. Psychol Health. 1998;14:1-24.

33. Lavsa SM, Holzworth A, Ansani NT. Selection of a validated scale for measuring medication adherence. J Am Pharm Assoc (2003). 2011;51:90-94.

34. Michetti P, Weinman J, Mrowietz U, Smolen J, PeyrinBiroulet L, Louis E, et al. Impact of Treatment-Related Beliefs on Medication Adherence in Immune-Mediated Inflammatory Diseases: Results of the Global ALIGN Study. Adv Ther. 2017;34:91-108.

35. de Vries ST, Keers JC, Visser R, de Zeeuw D, Haaijer-Ruskamp FM, Voorham J, et al. Medication beliefs, treatment complexity, and non-adherence to different drug classes in patients with type 2 diabetes. J Psychosom Res. 2014;76:134-138.

36. Foot H, La Caze A, Gujral G, Cottrell N. The necessityconcerns framework predicts adherence to medication in multiple illness conditions: A meta-analysis. Patient Educ Couns. 2016;99:706-717.

37. Anghel LA, Farcaş AM, Oprean RN. Medication adherence and persistence in patients with autoimmune rheumatic diseases: a narrative review. Patient Prefer Adherence. 2018;12:1151-1166. 